\title{
Des « certifficats \\ de virginité » aux \\ hyménoplasties \\ en France
}

> «Dans la République, on ne peut pas exiger des certificats de virginité pour se marier; dans la République, on ne doit jamais accepter que les lois de la religion puissent être supérieures aux lois de la République », telle est la déclaration en février 2020 du président Emmanuel Macron. Mais quel est donc le statut de ces certificats de virginité, est-il véritablement religieux ? Pourquoi se focaliser sur les « certificats de virginité » sans jamais évoquer la pratique concomitante de la réfection d'hymen? N'existe-t-il pas une diversité de pratiques chirurgicales participant du processus de revirginisation telle que la nymphoplastie ou la vaginoplastie; I'hyménoplastie ne serait-elle que la pointe émergée de l'iceberg ? <

«Vous allez bientôt vous marier? Peut-être qu'à l'approche du mariage, vous avez reçu une bague, vous êtes en train de choisir une robe, de commencer à dresser la liste de vos invités pour la fête? Mais pour d'autres, les préparatifs du mariage, ça ne ressemble pas à ça. Pour d'autres femmes, en France, en 2020, la fiancée est conduite chez un professionnel pour réaliser un test de virginité et repartir avec un certificat de virginité. Cette pratique, nous voulons y mettre fin... Les lois de la République sont supérieures à toute coutume, et la liberté des femmes ne se négocie pas », tel est le discours le $27 \mathrm{sep}-$ tembre 2020 de Marlène Schiappa, ministre chargée de la citoyenneté en France. Ce discours est inspiré de celui du président Emmanuel Macron tenu le 18 février 2020 à Mulhouse, à cette différence près que ce dernier se réfère non plus à la coutume mais à la religion: «Dans la République, on ne peut pas exiger des certificats de virginité pour se marier; dans la République, on ne doit jamais accepter que les lois de la religion puissent être supérieures aux lois de la République ».

Vignette (Photo, cellules déciduales de l'utérus @ Inserm/Le Goascogne, Claude).

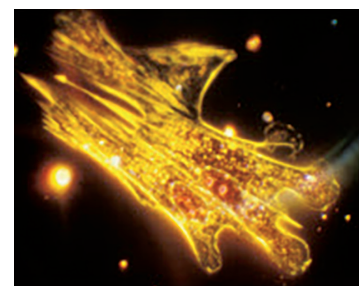

CNRS, Laboratoire d'anthropologie sociale (Collège de France), 52 rue du Cardinal Lemoine, 75005 Paris, France. corinne.fortier@college-de$\underline{\text { france.fr }}$

\section{Des certificats pas très musulmans}

Le ministre de l'Intérieur déclarait le 8 septembre 2020 : «Certains médecins osent encore certifier qu'une femme est vierge pour permettre un mariage religieux, malgré la condamnation de ces pratiques par le conseil de l'Ordre des médecins. On va non seulement l'interdire formellement, mais proposer la pénalisation ». II est ici fait référence à un lien entre exigence de virginité et mariage religieux, mais en dépit de ce que laisse penser l'expression même de « certificat de virginité », celle-ci ne constitue nullement une condition nécessaire au « certificat de mariage » en islam [1] et par ailleurs l'hymen ne saurait être une «preuve médico-légale » de virginité en France.

Car, même si les certificats de virginité en France concernent surtout des femmes musulmanes, cela ne signifie pas que cette pratique trouve sa source dans les textes islamiques; il n'est en effet jamais question d'hymen proprement dit en islam. Le Coran parle moins de virginité que de relations sexuelles illégitimes, et si l'impératif de virginité jusqu'au mariage reste très prégnant dans de nombreuses sociétés du petit Maghreb et du Moyen-Orient, dont la Turquie et l'Arabie Saoudite, et de l'Iran, l'Afghanistan, le Pakistan, il n'est pas uniquement présent chez les musulmans. II l'est aussi chez les Coptes en Égypte ou encore chez les chrétiens au Liban, témoignant que cet impératif est d'ordre culturel davantage que religieux.

À cet égard, l'injonction à garder sa virginité avant la nuit de noces est observable non seulement dans le monde arabe mais plus largement, jusqu'à récemment, dans le bassin méditerranéen [2], et ce quelle que soit la religion pratiquée, notamment dans l'Italie, l'Espagne, le Portugal catholiques, la Grèce orthodoxe, l'Arménie chrétienne, l'Albanie musulmane. 
L'exigence de virginité au mariage est donc avant tout une exigence culturelle, et elle n'est pas propre, par ailleurs, au bassin Méditerranée ou au monde arabe, puisqu'on la retrouve dans certains pays d'Asie, notamment, par le passé, en Chine [3], et encore récemment au Viêt$\operatorname{Nam}[4,5]$. Elle existe également en Afrique chez certains groupes du Niger, du Tchad, du Nigéria, du Cameroun, du Bénin, du Ghana, du Togo, du Gabon, et de Tanzanie ${ }^{1}$.

Elle est par ailleurs prônée ${ }^{2}$ par des mouvements évangéliques américains, qui aujourd'hui essaiment non seulement aux États-Unis, mais aussi dans certains pays africains ${ }^{3}$. Ainsi, en 2019, un rappeur américain défraya la chronique après avoir déclaré qu'il amenait chaque année sa fille de dix-huit ans chez un gynécologue pour vérifier sa virginité, ce qui conduisit l'État de New York à élaborer un projet de loi interdisant les certificats de virginité2.

\section{Des certificats intrusifs}

La présence d'un hymen anatomique imperforé est difficile à apprécier. Ambroise Paré (1510-1590), à la Renaissance, constatait que, de taille variable, l'hymen peut être naturellement perforé : «L'hymen est un repli membraneux plus ou moins circulaire, plus ou moins large, plus ou moins égal, quelquefois semi-lunaire, qui laisse une ouverture très petite dans les unes, et très grande dans les autres » [6] (p. 327). C'est donc le sang, censé survenir lors de la défloration, qui tient lieu de preuve visuelle de la virginité, même si ce signe est également très aléatoire, comme le signalait le naturaliste Buffon (1707-1788) au XVIII ${ }^{e}$ siècle dans son Histoire Naturelle: «Il est évident que l'effusion du sang, que l'on regarde comme une preuve réelle de virginité, ne se rencontre pas dans toutes les circonstances où l'entrée du vagin a pu être relâchée ou dilatée naturellement. Ainsi, toutes les filles, quoique non déflorées, ne répandent pas du sang... » [6] (p. 329).

Si le sang de la défloration demeure toujours important de nos jours dans nombre de sociétés, le certificat médical permet d'attester de la virginité avant la nuit de noces, y compris quand précisément la jeune femme n'est plus vierge. Preuve de la médicalisation croissante des sociétés, les médecins se retrouvent à présent sollicités pour délivrer des certificats de virginité. Ils ont désormais pris la place des matrones, des mères, ou des hommes de religion ${ }^{4}$, qui pouvaient constater la virginité d'une femme par l'examen de son hymen.

$\mathrm{Si}$, aujourd'hui, l'examen gynécologique pratiqué dans le cadre des certificats de virginité est médicalisé, cet examen s'apparente à un viol de l'intimité, comme le rappelait en 2007 le conseil national de l'Ordre des médecins : "Le conseil national de l'Ordre des médecins

1 Musabyimana G. Les paradoxes culturels : virginité et défloration rituelle en Afrique. Échosd'Afrique. com, 5 décembre 2019, http://www.echosdafrique.com/20191205-6036.

2 « Tests de virginité : New York va examiner un projet de loi », La presse, 3 décembre 2019, https:// www.lapresse.ca/international/etats-unis/2019-12-03/tests-de-virginite-new-york-va-examiner-unprojet-de-loi.

3 Ben Mahmoud F. Like a Virgin. La virginité féminine fait de la résistance. Documentaire, 2020.

${ }^{4}$ C'est le cas par exemple au Congo aujourd'hui, où des pasteurs évangéliques, avant de « bénir le mariage », dénudent la mariée, et procèdent devant l'assistance, à un toucher vaginal afin de s'assurer de sa virginité. Boukaka B. Congo - Religion : Quand le pasteur vérifie la virginité de la mariée avant de bénir I'union. Les Échos du Congo Brazaville, 28 août 2018, https://lesechos-congobrazza.com/societe/5125congo-religion-quand-le-pasteur-s-assure-de-la-virginite-de-la-mariee-avant-de-benir-l-union. considère que, n'ayant aucune justification médicale et constituant une violation du respect de la personnalité et de l'intimité de la jeune femme (notamment mineure) contrainte par son entourage de s'y soumettre, un tel examen ne relève pas du rôle du médecin. Celui-ci doit donc refuser l'examen et la rédaction d'un tel certificat qui nous paraît contraire à la dignité de la femme » [7].

\section{Des certificats « policiers »?}

Il existe une inégalité du point de vue du genre au regard de l'exigence de virginité, contrôle qui se focalise sur cet élément anatomique proprement féminin qu'est l'hymen. Mais cette surveillance particulière des femmes ne s'explique pas par le fait qu'elles possèdent un hymen, mais par une autre donnée biologique différentielle entre les sexes bien plus déterminante, à savoir que les enfants se développant dans le corps féminin, les sociétés cherchent à prévenir le risque d'une naissance illégitime en contrôlant la sexualité des femmes.

L'expression « certificat de virginité » accorde une valeur scientifique à un examen qui renseigne moins sur l'hymen lui-même que sur les représentations qui lui sont attribuées, représentations qualifiées déjà d'« imaginaires » [6] au xvI siècle par Ambroise Paré, et, au XVIII ${ }^{e}$ siècle, de « chimériques » par Buffon [6], et de «pseudo-scientifiques, imaginaires et policières » [8] par Diderot (1713-1784) et d'Alembert (1717-1783) dans la rubrique « virginité » de l'Encyclopédie.

Aujourd'hui, les certificats de virginité s'apparentent d'une certaine manière aux tests de virginité effectués par la police dans des pays comme l'Égypte, lors des révoltes de 2011 où ils étaient imposés aux femmes osant manifester dans l'espace public réservé aux hommes [9]. Les certificats de ce type ont pour but de distinguer deux catégories de femmes, celles vertueuses qui ont conservé l'intégrité de leur hymen, et celles dépravées qui l'ont perdue, confortant ainsi la représentation binaire de la femme : la vierge ou la putain.

\section{Hymens complaisants et certificats patriarcaux}

Il est à cet égard significatif que beaucoup de femmes - la moitié selon les gynécologues obstétriciens ne déchirent pas leur hymen lors des premiers rapports sexuels ${ }^{5}$. L'expression «d'hymen complaisant », employée à ce propos, y compris par le conseil national

${ }^{5}$ Comme l'explique le dictionnaire médical de 2020 de l'Académie de médecine: «À partir de la puberté, l'hymen peut être suffisamment souple et élastique pour permettre des rapports sexuels complets sans déterminer de lésion traumatique ». http://dictionnaire.academie-medecine.fr/aecfepkhje.html?q=caroncules $\% 20$ myrtiformes. 
de l'Ordre des médecins [7], véhicule un jugement moral où la femme est implicitement identifiée à son hymen. En effet, n'est-ce-pas la femme elle-même qui est jugée « complaisante », coupable de se livrer à des rapports sexuels auxquels elle semble prendre plaisir puisque son hymen ne se rompt pas, comme s'il se dérobait à fournir la preuve anatomique de ces plaisirs défendus.

Pour beaucoup de femmes aujourd'hui, la virginité est un sujet très intime faisant partie de leur vie privée, qui ne devrait concerner personne d'autre qu'elles-mêmes, et par conséquent ne faire en aucun cas l'objet d'un certificat médical, rédigé par un tiers, fût-il gynécologue, et adressé à d'autres tiers, comme leur famille, leur future belle-famille et leur futur époux. Bien que certains médecins s'en défendent, affirmant aider les femmes en leur délivrant un tel certificat, ils accréditent plutôt ainsi l'injonction faite aux femmes de livrer des preuves de leur virginité, injonction qui vient cautionner le contrôle masculin exercé sur leur corps.

Les médecins qui rédigent un certificat de virginité, fût-il « de complaisance », tissent de fait une complicité souterraine avec la famille de la femme, ainsi qu'avec son futur époux et la famille de ce dernier. L'établissement de certificats de virginité vient corroborer l'idée que le corps de la femme ne lui appartient pas, mais est la propriété de sa famille avant de devenir celle de son époux. Plutôt que de s'attaquer à cette norme patriarcale en soutenant le droit des femmes à disposer de leur corps, ils contribuent au contrôle social opéré sur elles, légitimant des pratiques qui s'opposent à leur autonomisation.

Ainsi que le déclarait Marlène Schiappa dans sa tribune du 26 septembre 2020 : «Nous l'affirmons sans craindre de choquer : cette pratique, qui bafoue la liberté de disposer de son propre corps, est une atteinte majeure à la dignité des femmes... Établir des certificats de virginité sous prétexte que cela protégerait des filles de violences familiales, c'est tolérer cette tradition néfaste et accepter son maintien ${ }^{6}$.

L'évolution des rapports de genre permet en outre de penser qu'en France, comme dans les pays arabes, la norme de virginité est amenée à disparaître à plus ou moins long terme, dans la mesure où elle apparaît difficilement compatible avec les aspirations des jeunes générations à la sexualité [10].

\section{De l'excision aux certificats}

Le projet de loi en France pénalisant les médecins qui délivrent des certificats de virginité est en cohérence avec la lutte internationale pour «l'éradication des tests de virginité » (Eliminating Virginity Testing) entreprise en 2018 par l'Organisation mondiale de la santé (OMS), afin d'interdire ces tests «médicalement inutiles, humiliants et traumatisants », qui constituent une «violation des droits fondamentaux des femmes et des filles ${ }^{7}$.

${ }^{6}$ Cette tribune a été écrite dans le Journal du Dimanche par Marlène Schiappa ainsi que par quatre cosignataires : Chrysoula Zacharopoulou, médecin, députée européenne, Hubert Sage, médecin, Michèle Vianès, présidente de l'association « Regards de femmes », Assia Benziane, maire adjointe aux droits des femmes de Fontenay-sous-Bois. Schiappa M. Abolissons les certificats de virginité ! https://www.lejdd.fr/ Societe/tribune-marlene-schiappa-abolissons-les-certificats-de-virginite-3994406.

${ }^{7}$ Eliminating virginity testing: an interagency statement. World Health Organization, 2018, https://apps. who.int/iris/bitstream/handle/10665/275451/WHO-RHR-18.15-eng.pdf?ua=1.
Déjà en 2003, le conseil national de l'Ordre des médecins français s'opposait à cette pratique jugée « barbare »: «Nous sommes résolument opposés aux tests de virginité. C'est une pratique barbare, rétrograde et totalement sexiste $\gg^{8}$, tandis que Marlène Schiappa la qualifie d'《ancienne » et d' « obscurantiste » : «C'est une pratique ancienne qui ne respecte pas la dignité du corps des femmes. Aucune citoyenne en France n'a à justifier de sa virginité vis-à-vis de son fiancé, vis-àvis de sa famille, ou vis-à-vis de qui que ce soit. Comme il y a des années la République française a dit non et stop à l'excision, aujourd'hui la République française prend ses responsabilités et veut mettre fin à la pratique des certificats de virginité... $»^{6}$.

Comme on le voit, la lutte contre les certificats de virginité en France est assimilée à celle contre l'excision, à la différence près que, dans les années 1980, les « professionnels » pénalisés étaient les exciseuses, tandis que, aujourd'hui, ce sont les médecins. Ces deux types de «professionnels », bien que profondément dissemblables, sont ici mis sur le même plan, une telle comparaison présentant l'avantage de mettre fin à la hiérarchie entre les actes médicaux « civilisés et progressistes » et les rituels «barbares d'un autre âge » [11].

\section{Certificats et hyménoplasties}

Les « certificats de virginité » du médecin ne remplacent pas néanmoins la preuve par le sang de la défloration. Ainsi, la réparation « de papier » qu'offre les « certificats», fussent-ils de complaisance, n'empêche pas les femmes de procéder à une reconstruction « charnelle » de leur hymen, celle-ci précédant bien souvent celle-là, dans le but de «saigner »lors de leur nuit de noces. Aujourd'hui, en effet, les techniques chirurgicales d'hyménoplastie permettent de se refaire une virginité.

Le rôle revirginisateur de l'hyménoplastie se retrouve dans d'autres chirurgies sexuelles pratiquées en France telles que la nymphoplastie, un nom qui renvoie à la figure de la nymphe, soit une jeune fille vierge. Elle est également nommée plus prosaïquement labiaplastie puisque, comme dans l'excision [12], elle consiste à couper une partie des petites lèvres, mais à la différence de cette dernière, elle est non seulement légale mais partiellement remboursée par l'Assurance maladie. Elle est réalisée par des femmes qui ont le sentiment que leurs lèvres sont «trop » visibles eu égard

8 Bisseul S. L'hyménoplastie, le droit et l'éthique. Legavox.fr, 10 juillet 2020,
https://www.legavox.fr/blog/stella-bisseuil/hymenoplastie-droit-ethique-21517. htm. 
au dictat des représentations publicitaires ou pornographiques qui donnent une image lisse et sans relief de la vulve, ressemblant à un sexe virginal de petite fille.

Une autre chirurgie revirginisatrice comparable à l'hyménoplastie est la vaginoplastie, suggestivement nommée « rajeunissement vaginal ». Également remboursée par l'Assurance maladie, elle resserre les parois vaginales afin de retrouver un sexe de jeune fille d'avant la perte de virginité. Jusque dans les années 1990, il n'était pas rare en France que des médecins l'accomplissent sur des femmes venant d'accoucher, recousant leur vagin de manière «plus serrée » afin que leur époux soit sexuellement satisfait, pratique significativement appelée «le point du mari » [13].

\section{Conclusion}

On peut se demander pourquoi le nouveau projet de loi ne se focalise que sur les « certificats de virginité » sans jamais évoquer la pratique de l'hyménoplastie qui représente tout autant sinon davantage une violence à l'égard des femmes. Ce point aveugle tient à ce qu'il est plus facile de pénaliser des gynécologues qui délivrent des certificats de virginité, le plus souvent des femmes travaillant dans le public, que des chirurgiens plasticiens qui réalisent des hyménoplasties, le plus souvent des hommes opérant dans le privé.

Mais surtout s'attaquer à cette pratique reviendrait à ouvrir la boîte de Pandore des opérations esthétiques, et à faire prendre conscience du continuum existant entre ces chirurgies, notamment entre les chirurgies sexuelles que sont l'hyménoplastie, la nymphoplastie et la vaginoplastie. Compte tenu de la proximité entre ces opérations qui ont pour finalité commune la revirginisation du corps féminin, interdire l'une devrait conduire à interdire les autres.

La seule différence établie officiellement entre ces opérations est celle d'une altérité culturelle et religieuse, l'hyménoplastie s'adressant à des femmes «musulmanes » prétendument soumises et opprimées, tandis que les autres opérations concernent des femmes supposées libres et autonomes. Or, il nous semble nécessaire de se départir de l'exclusive consistant à se focaliser sur les demandes de revirginisation de «musulmanes » décrites comme «obscurantistes », tout en semblant ignorer que des femmes présumées «émancipées 》 ont recours à des opérations analogues.

Cette perspective permettrait de dépasser le clivage ethnocentrique entre «nous » et «les autres » afin de poser la question plus générale de l'aliénation du corps féminin, aliénation à laquelle certains actes médicaux ressortant de la chirurgie esthétique ou réparatrice participent, sans pour autant faire l'objet d'une quelconque pénalisation, interdiction ou mise en perspective critique.

\section{SUMMARY}

From virginity certificates to hymenoplasty surgery in France

"In the Republic, it is not allowed to deliver virginity certificates, prior to marriage; in the French Republic, the laws of religion cannot prevail over the laws of the Republic" said President Emmanuel Macron in February 2020. Nevertheless, what about the religious status of these certificates? Why focusing only on virginity certificates without mentioning the concomitant practice of hymen rehabilitation? There is a whole range of revirginization surgical practices, such as nymphoplasty or vaginoplasty, among which hymenoplasty figures only incidentally?

\section{LIENS D'INTÉRÊT}

L'auteure déclare n'avoir aucun lien d'intérêt concernant les données publiées dans cet article.

\section{RÉFÉRENCES}

1. Fortier C. Le droit musulman en pratique : genre, filiation et bioéthique. In : Fortier C, ed. Actualités du droit musulman : genre, filiation et bioéthique. Droit et Cultures $2010 ; 59$ : 11-40. http://droitcultures.revues.org/1923.

2. Tillion G. Le Harem et les cousins. Paris : Seuil, 1966.

3. Van Gulick R. La vie sexuelle dans la Chine ancienne. Paris : Gallimard, 1977.

4. Dinh TH. Vraies et fausses vierges au Viêt Nam. La falsification corporelle en question. Extrême-Orient $2010 ; 32: 163-91$.

5. Zavala de Cosio ME, De Loenzien M, Luu Bich N. Mutations démographiques et sociales du Vietnam contemporain. Nanterre : Presses Universitaires de Paris Ouest, 2016.

6. Panckoucke (J), Agasse H, Agasse TC, Vicq-Dazyr F. Encyclopédie méthodique : médecine. Paris : Panckoucke, 1792.

7. Lansac J. Faut-il faire des certificats de virginité ? Brèves du Collège, Conseil national des obstétriciens et gynécologues de France 2007 ; 13. http://www.cngof.net/Publications-CNGOF/Breves/Breves/CNGOFbreves-13.pdf.

8. Sissa J. Le corps virginal. Paris : Vrin, 1987.

9. Fortier C, Monqid S. Le corps féminin en contexte arabo-musulman : entre autonomisation et domination. In : Fortier C, Monqid S, eds, Corps des femmes et espaces genrés arabo-musulmans. Paris : Karthala, 2017 : 9-19.

10. Fortier C, Kreil A, Maffi I. Introduction. Reinventing love? Gender, intimacy and romance in the Arab world. In : Fortier C, Kreil A, Maffi I, eds. Reinventing love? Gender, intimacy and romance in the Arab World. Berne : Peter Lang (Middle East, social and cultural studies-Études culturelles et sociales sur le Moyen-Orient), 2018:9-32.

11. Fortier C. Réparer les corps et les sexes. Des rituels sexués aux chirurgies sexuelles. In : Fortier C, ed. Réparer les corps et les sexes, vol. 1. Excision, circoncision, et reconstruction clitoridienne. Droits et Cultures $2020 ; 79$ : 9-14. https://journals.openedition.org/droitcultures/5973.

12. Fortier C. Sculpter la différence des sexes. Excision, circoncision et angoisse de castration (Mauritanie). In : Lachheb M, ed., Penser le corps au Maghreb. Paris : Karthala/IRMC (Hommes et Sociétés), 2012 : 35-66.

13. Fortier C. Reconstruction clitoridienne, excision et circoncision. Variations autour d'un sexe féminin phallique. In Fortier C, ed. Réparer les corps et les sexes, vol. 1. Excision, circoncision, et reconstruction clitoridienne. Droits et Cultures $2020 ; 79: 29-76$. https://journals.openedition.org/droitcultures/5977.

14. Fortier C. Seins, reconstruction, et féminité. Quand les Amazones s'exposent. In Fortier C, ed. Réparer les corps et les sexes, vol. 2. Intersexuation, transidentité, reconstruction mammaire, et surdité. Droits et Cultures 2020 ; 80 : 53-74. https://journals.openedition.org/droitcultures/6721.
TIRÉS À PART

C. Fortier 